\title{
ON THE GLOBAL EXISTENCE OF SOLUTIONS OF A REACTION-DIFFUSION EQUATION WITH EXPONENTIAL NONLINEARITY
}

\author{
ASSIA BARABANOVA \\ (Communicated by Barbara Lee Keyfitz)
}

\begin{abstract}
We generalize the result of Haraux and Youkana concerning the global existence of nonnegative solutions of a reaction-diffusion equation with exponential nonlinearity. We also show the asymptotic behavior of the global solutions as $t \rightarrow \infty$.
\end{abstract}

\section{INTRODUCTION}

In this paper we consider the reaction-diffusion system

$$
\begin{cases}\frac{\partial u}{\partial t}-d_{1} \Delta u+u \varphi(v)=0 & \text { on } \mathbb{R}^{+} \times \Omega, \\ \frac{\partial v}{\partial t}-d_{2} \Delta v-u \varphi(v)=0 & \text { on } \mathbb{R}^{+} \times \Omega,\end{cases}
$$

where $\Omega$ is an open, bounded domain in $\mathbb{R}^{n}$ of class $C^{1}, d_{1}, d_{2}$ are positive constants, and $\varphi$ is a nonnegative function of class $C^{1}\left(\mathbb{R}^{+}\right)$.

We assume that the solutions of (1) also satisfy the homogeneous boundary conditions

$$
\begin{cases}\eta_{1} \frac{\partial u}{\partial n}+\left(1-\eta_{1}\right) u=0 & \text { on } \mathbb{R}^{+} \times \Gamma, \\ \eta_{2} \frac{\partial v}{\partial n}+\left(1-\eta_{2}\right) v=0 & \text { on } \mathbb{R}^{+} \times \Gamma,\end{cases}
$$

where $\Gamma=\partial \Omega$ and $\eta_{1}(x), \eta_{2}(x)$ are nonnegative functions of class $C^{1}(\Gamma)$ with $\eta_{1}, \eta_{2} \leq 1$.

We study the question of the existence of global solutions of problem (1)-(2) in the class $C(\overline{\mathbf{\Omega}})$ with initial data

$$
u(x, 0)=u_{0}, \quad v(x, 0)=v_{0},
$$

where $u_{0}, v_{0}$ are of class $L^{\infty}(\Omega)$ and $u_{0}, v_{0} \geq 0$.

When $d_{1}=d_{2}$ the existence of global solutions of (1)-(3) is an evident consequence of the maximum principle.

This problem was initially posed by Martin and has attracted the attention of many authors. First, Alikakos [1] established the existence of global solutions

Received by the editors July 20, 1992 and, in revised form, January 18, 1993 and March 1, 1993.

1991 Mathematics Subject Classification. Primary 35K57. 
when $\varphi(v) \leq C\left(1+|v|^{n+2 / n}\right)$. Then, Masuda [6] obtained a positive result for the case $\varphi(v) \leq C\left(1+|v|^{\beta}\right)$ with arbitrary $\beta>0$. The best success in this direction was achieved by Haraux and Youkana [4]. They proved the existence of global solutions for $\varphi(v)=e^{\alpha v^{\beta}}, 0<\beta<1, \alpha>0$. In the case $\beta=1$ some restrictions on $u_{0}$ appear. Youkana in [7] treated this case in small dimensions $n=1$ or 2 and obtained the existence of global solutions with the condition

$$
\left\|u_{0}\right\|_{L^{\infty}(\Omega)} \leq \frac{d_{1}}{\alpha\left(d_{1}+d_{2}\right)} .
$$

The proof of Youkana is based on Sobolev's embedding theorem which is possible to apply in this problem only for dimensions $n=1,2$.

Here we analyze this problem using semigroup methods and Lyapunov function techniques. We show that for

$$
\varphi(v) \leq e^{\alpha v}
$$

and for arbitrary dimension $n$, solutions of (1)-(3) are global for arbitrary $v_{0}$ and $u_{0}$ satisfying

$$
\left\|u_{0}\right\|_{L^{\infty}(\Omega)}<\frac{8 d_{1} d_{2}}{\alpha n\left(d_{1}-d_{2}\right)^{2}} .
$$

We remark that the restriction (5) is weak when $d_{1}$ is close to $d_{2}$.

In conclusion, we observe that the problem is still open when $\varphi$ has faster growth than (4) or when (5) does not hold.

\section{STATEMENT AND PROOF OF THE MAIN RESULT}

We denote by $\|\cdot\|_{p}$ the norm in $L^{p}(\Omega), 1 \leq p \leq \infty$.

For nonnegative $u_{0}$ and $v_{0}$ there exists a local nonnegative solution $(u, v)$ of class $C(\bar{\Omega})$ of (1)-(3) on ]0,T[, where $T$ is the eventual blowing-up time in $L^{\infty}(\Omega)$ [2]. It is evident that $u$ satisfies the maximum principle, i.e., $\|u(t)\|_{\infty} \leq$ $\left\|u_{0}\right\|_{\infty}$. From the results of [3] it follows that it is sufficient to get a uniform estimate for $\|u \varphi(v)\|_{p}$ on $] 0, T$ [ for some $p>n / 2$ in order to establish the existence of global solutions (see also [5]).

The main result of this paper is

Theorem 1. Assume $\varphi(v) \leq e^{\alpha v}$. Then the solutions of (1)-(3) with nonnegative initial data $u_{0}, v_{0}$ in $L^{\infty}(\Omega)$ satisfying (5) are global and uniformly bounded on $] 0 ; \infty[\times \Omega$.

To prove this theorem we need the following

Proposition 2. Assume that (4) holds and let $(u, v)$ be a solution of (1)-(3) with arbitrary $v_{0}$ and $u_{0}$ satisfying (5). Let

$$
g(u)=\left(\frac{C}{C-u}\right)^{4 d_{1} d_{2} /\left(d_{1}-d_{2}\right)^{2}} .
$$

Then there exists $C>\left\|u_{0}\right\|_{\infty}$ and $p>n / 2$ such that

$$
\int_{\Omega} g(u) e^{\alpha p v} d x
$$

is nonincreasing on $] 0, T[$. 
Since $g(u) \geq 1$, we observe

$$
\left(\|\varphi(v)\|_{p}\right)^{p}=\int_{\Omega}|\varphi(v)|^{p} d x \leq \int_{\Omega} e^{\alpha p v} d x \leq \int_{\Omega} g(u) e^{\alpha p v} d x .
$$

By Proposition 2 we deduce

$$
\left(\|\varphi(v)\|_{p}\right)^{p} \leq \int_{\Omega} g(u) e^{\alpha p v_{0}} d x \leq|\Omega| g\left(\left\|u_{0}\right\|_{\infty}\right) e^{\alpha p\left\|v_{0}\right\|_{\infty}} .
$$

Hence $u \varphi(v)$ is uniformly bounded in $L^{p}(\Omega)$ for all $t \in[0 ; T]$. Using the results of [3] we conclude that the solutions of $(1)-(3)$ are global and uniformly bounded on $] 0 ; \infty[\times \Omega$.

\section{ASYMPTOTIC BEHAVIOR AS $t \rightarrow+\infty$}

The behavior of nonnegative global solutions of (1)-(3) as $t \rightarrow+\infty$ is treated in papers $[4,6]$. In particular, it is proved that there exist two nonnegative constants $u^{*}, v^{*}$ such that

$$
\left\|u(t)-u^{*}\right\|_{\infty} \rightarrow 0, \quad\left\|v(t)-v^{*}\right\|_{\infty} \rightarrow 0,
$$

and $u^{*} \varphi\left(v^{*}\right)=0$. It is evident that $u^{*}=v^{*}=0$ when both $u$ and $v$ have nonNeumann boundary conditions. The following theorem describes the asymptotic behavior of solutions with $\varphi(v)=e^{\alpha v}$.

Theorem 3. Let $(u, v)$ be any nonnegative global solution of (1)-(3) with $\eta_{1}$, $\eta_{2} \neq 1$.

Let $\lambda_{i}$ be the first eigenvalue of $-\Delta$ in $\Omega$ with boundary condition (2) defined by $\eta_{i}, i=1,2$.

Then there exist constants $C_{1}, C_{2}, C_{3}>0$ such that

$$
\begin{gathered}
\|u(t)\|_{\infty} \leq C_{1} e^{-\left(\lambda_{1} d_{1}+1\right) t}, \\
\|v(t)\|_{\infty} \leq C_{2} e^{-\min \left\{\left(\lambda_{1} d_{1}+1\right), \lambda_{2} d_{2}\right\} t} \quad \text { if }\left(\lambda_{1} d_{1}+1\right) \neq \lambda_{2} d_{2}, \\
\|v(t)\|_{\infty} \leq\left(C_{2}+C_{3} t\right) e^{-\lambda_{2} d_{2} t} \quad \text { if }\left(\lambda_{1} d_{1}+1\right)=\lambda_{2} d_{2} .
\end{gathered}
$$

Proof. The solution of the equation

$$
\frac{\partial u}{\partial t}-d_{1} \Delta u+u=0
$$

with boundary condition (2) and initial data (3) is a supersolution for

$$
\frac{\partial u}{\partial t}-d_{1} \Delta u+u e^{\alpha v}=0
$$

with the same boundary condition and initial data. It is well known from [2] that the solution of the linear equation (12) has asymptotics $e^{-\left(\lambda_{1} d_{1}+1\right) t}$ so that we have proven statement (9).

Since $\|v(t)\|_{\infty}$ is bounded, there exists a constant $C$ such that $u e^{\alpha v} \leq$ $C e^{-\left(\lambda_{1} d_{1}+1\right) t}$. Applying the expression of the solution of equation (1) in terms of the semigroup generated by $\frac{\partial}{\partial t}-d_{2} \Delta$, as in $[2,3]$, we obtain the inequality

$$
\|v(t)\|_{\infty} \leq M_{1} e^{-\lambda_{2} d_{2} t}+M_{2} e^{-\lambda_{2} d_{2} t} \int_{0}^{t} e^{\left(-\left(\lambda_{1} d_{1}+1\right)+\lambda_{2} d_{2}\right) \sigma} d \sigma,
$$

where $M_{1}, M_{2}>0$. 
Let us set $\lambda=\lambda_{1} d_{1}+1$ and $\mu=\lambda_{2} d_{2}$. If $\mu \neq \lambda$, then from (13) we get

$$
\begin{gathered}
\|v(t)\|_{\infty} \leq M_{1} e^{-\mu t}+\frac{M_{2}}{\mu-\lambda} e^{-\mu t}\left(e^{(\mu-\lambda) t}-1\right) \\
=\left(M_{1}-\frac{M_{2}}{\mu-\lambda}\right) e^{-\mu t}+\frac{M_{2}}{\mu-\lambda} e^{-\lambda t} .
\end{gathered}
$$

If $\mu \leq \lambda$ we have

$$
\|v(t)\|_{\infty} \leq\left(M_{1}+\frac{M_{2}}{\lambda-\mu}\right) e^{-\mu t} \leq C_{2} e^{-\mu t} .
$$

If $\mu \geq \lambda$, then

$$
\|v(t)\|_{\infty} \leq M_{1} e^{-\mu t}+\frac{M_{2}}{\mu-\lambda} e^{-\lambda t} \leq C_{2} e^{-\lambda t},
$$

which proves statement (10).

Let $\mu=\lambda$. In this case resonance appears and we have

$$
\|v(t)\|_{\infty} \leq M_{1} e^{-\mu t}+M_{2} t e^{-\mu t}=\left(M_{1}+M_{2} t\right) e^{-\mu t},
$$

so the proof of the theorem is complete.

\section{ACKNOWLEDGMENT}

I would like to thank Professors A. Haraux and T. Cazenave for their kind advice.

\section{REFERENCES}

1. N. D. Alikakos, $L^{p}$-bounds of solutions of reaction-diffusion equations, Comm. Partial Differential Equations 4 (1979), 827-868.

2. T. Cazenave and A. Haraux, Introduction aux problèmes d'évolution semilinéaires, Mathématiques \& Applications, vol. 1, Ellipses, Paris, 1990.

3. A. Haraux and M. Kirane, Estimations $C^{1}$ pour des problèmes paraboliques non-linéaires, Ann. Fac. Sci. Toulouse 5 (1983), 265-280.

4. A. Haraux and A. Youkana, On a result of $K$. Mazuda concerning reaction-diffusion equations, Tôhoku Math. J. 40 (1988), 159-163.

5. D. Henry, Geometric theory of semilinear parabolic equations, Lecture Notes in Math., vol. 840, Springer-Verlag, Berlin and New York, 1981.

6. K. Masuda, On the global existence and asymptotic behavior of reaction-diffusion equations, Hokkaido Math. J. 12 (1983), 360-370.

7. A. Youkana, Thèse de 3ème cycle, Chapitre 3, Université Paris 6, 1986.

Departement de Mathematiques et d'Informatique, Ecole Normale Superieure, 45 RUE D'Ulm, 75005 Paris, France

Current address: Department of Mathematics, Faculty of Science, 632 Central Academic Building, University of Alberta, Edmonton, Canada T6G 2G1

E-mail address: abaraban@vega.math.ualberta.ca 